\title{
Neurotrophin-3 Reverses Chronic Mechanical Hyperalgesia Induced by Intramuscular Acid Injection
}

\author{
Rohan Gandhi, Janelle M. Ryals, and Douglas E. Wright \\ Department of Anatomy and Cell Biology, University of Kansas Medical Center, Kansas City, Kansas 66160
}

\begin{abstract}
Injection of acid into the gastrocnemius muscle results in a persistent, mechanical hyperalgesia of the hindpaw (Sluka et al., 2001). Here, the ability of neurotrophins to alter the development of this secondary hyperalgesia was assessed using transgenic mice and exogenous neurotrophin administration. Acid-induced hyperalgesia was measured in wild-type and transgenic mice that overexpress neurotrophin-3 (NT-3) in muscle (myo/NT-3 mice). Mechanical and thermal sensitivity of the hindpaws were assessed after injections of acidic saline, $\mathrm{pH} 4$, into the right medial gastrocnemius. Wild-type mice exhibited mechanical but not thermal hyperalgesia in both paws $1 \mathrm{~d}$ after acid injection. In contrast, myo/NT-3 mice developed a transient mechanical hyperalgesia in both paws that disappeared by 2-3 $\mathrm{d}$. The reversal of hyperalgesia in myo/NT-3 mice could be mimicked by intramuscular administration of exogenous NT-3 to acid-injected mice but not by other neurotrophins. The route of NT-3 administration appears critical, because intrathecal or intraperitoneal delivery were ineffective. The hyperalgesia could only be reversed by NT-3 treatment concurrent with acid injection and not after the emergence of hyperalgesia. The acid-induced hyperalgesia did not redevelop after the termination of NT-3 treatment, suggesting that NT-3 permanently reversed the hyperalgesia. Consistent with the behavioral data, paw palpation of acid-injected mice significantly increased Fos expression in the spinal cord of wild-type but not myo/NT-3 or NT-3-injected mice. The attenuation of hyperalgesia suggests that NT-3 may be a modulator of muscle-derived pain, and NT-3 may suppress events that lead to secondary hyperalgesia triggered by insult to muscle afferents.
\end{abstract}

Key words: transgenic mice; neurotrophin-3; pain; muscle; hypersensitivity; Fos

\section{Introduction}

The mechanisms underlying pain derived from musculoskeletal origin remain poorly understood (Graven-Nielsen and Mense, 2001). Recently, animal models have been developed to simulate muscle-derived chronic pain (Kehl et al., 2000; Sluka et al., 2001; Radhakrishnan et al., 2003; Schafers et al., 2003). One in particular uses acid injection into the gastrocnemius to produce a persistent mechanical, but not thermal, hyperalgesia of the hindpaw (Sluka et al., 2001). Two acid injections over several days are required, and the secondary hyperalgesia spreads to both paws. Evidence suggests that central mechanisms contribute to the development and maintenance of the hyperalgesia (Sluka et al., 2001, 2002; Skyba et al., 2002; Hoeger-Bement and Sluka, 2003). Mice deficient in the acid-sensing ion channel-3 (ASIC3) fail to develop the acid-induced hyperalgesia, implicating this ion channel in this response (Price et al., 2001).

Aside from the involvement of ASIC3, the peripheral mechanisms or the afferent fiber types involved in the development of acid-induced chronic pain are not known. Skeletal muscles are

Received Nov. 24, 2003; revised Sept. 10, 2004; accepted Sept. 14, 2004.

This work was supported by National Institutes of Health Grants R21NS38844 and P01DE07734 (D.E.W.). We thank K. E. McCarson for technical assistance and members of the Wright laboratory for helpful comments during the preparation of this manuscript.

Correspondence should be addressed to Douglas Wright, Department of Anatomy and Cell Biology, University of Kansas Medical Center, Kansas City, KS 66160. E-mail: dwright@kumc.edu.

DOI:10.1523/JNEUROSCI.0899-04.2004

Copyright $\odot 2004$ Society for Neuroscience $\quad$ 0270-6474/04/249405-09\$15.00/0 innervated by a range of sensory axons that include $\mathrm{A} \alpha, \mathrm{A} \beta, \mathrm{A} \delta$, and C-type afferent fibers. In rats, $60 \%$ of muscle afferents are myelinated, and the remaining $40 \%$ are unmyelinated (Lawson, 1992; Graven-Nielsen and Mense, 2001). Central projections from these afferents terminate in nearly all spinal cord laminas (Fyffe, 1992). The diversity of muscle afferent fiber types ensures that a wide range of spinal neurons receive input from muscle afferents.

The neurotrophins are a family of secreted factors that include nerve growth factor (NGF), brain-derived neurotrophic factor (BDNF), neurotrophin-3 (NT-3), and neurotrophin-4/5 (NT-4/ $5)$. Each has been shown to exert trophic activity on distinct populations of dorsal root ganglion (DRG) neurons. The NGFrelated neurotrophins signal via tyrosine kinase (Trk) receptors and bind a common receptor with low affinity (p75). The TrkA receptor selectively binds NGF, TrkB binds BDNF and NT-4/5, and TrkC shows highest affinity for NT-3 (Snider, 1994). Glial cell line-derived neurotrophic factor (GDNF) also exerts trophic activity on DRG neurons (Molliver et al., 1997). All of these neurotrophins are expressed in muscle (Nagano and Suzuki, 2003). However, the majority (75\%) of muscle afferents express TrkC and are responsive to NT-3, whereas 20 and 50\% express receptors for and are responsive to NGF and BDNF, respectively (McMahon et al., 1994). Although NT-3 supports large muscle afferents, there is evidence that TrkC may be expressed by $\mathrm{C}$ and/or $\mathrm{A} \delta$ fibers as well (McMahon et al., 1994).

Here, we addressed whether members of the neurotrophin 
family can alleviate the mechanical hyperalgesia caused by intramuscular acid injection using transgenic mice and exogenous neurotrophin administration. Our results identify NT-3 as a powerful modulator of hyperalgesia evoked by intramuscular acid injection. The neuroprotection is unique to NT-3, is most effective when NT-3 is accessed from within the muscle, and appears permanent after the cessation of NT-3 treatment. Together, these results suggest that NT-3 is a significant modulator of muscle pain and can suppress events that lead to persistent hyperalgesia induced by insult to muscle afferents.

\section{Materials and Methods Animals}

All research performed conformed to National Institutes of Health guidelines in accordance with the guidelines specified by the University of Kansas Medical Center Animal Care and Use Protocol. Myo/NT-3 mice were generated as described previously, and a breeding colony has been established (Wright et al., 1997). These mice have been bred to a CF-1 background for over 10 generations, and genotypes for all transgenic mice were determined by PCR. Hemizygous males and females were bred to generate litters containing wild-type and transgenic mice so that all comparisons could be made among littermates. Both male and female myo/NT-3 mice were used. In addition to the transgenic mice, 2-month-old CF-1 male mice $(n=121)$ were purchased from Charles River (Wilmington, MA) and housed under specific pathogen-free conditions in the Laboratory Animal Resources at the University of Kansas Medical Center. CF-1 mice were chosen to match the genetic background of the transgenic myo/NT-3 mice. All mice were provided water and food ad libitum.

\section{Acid injection}

All mice receiving injections of acidic saline ( $20 \mu \mathrm{l}$ of acidic saline, $\mathrm{pH} 4$ ) were injected on days 0 and 2 in the right gastrocnemius muscle. Injections were made with a $1 \mathrm{ml}$ latex-free insulin syringe with $10 \mu \mathrm{l}$ increments (Becton Dickinson, Franklin Lakes, NJ).

\section{Trophic factor administration}

Groups of acid-injected mice $(n=8)$ were treated with NT-3 ( $1 \mu \mathrm{g}$ in 5 $\mu \mathrm{l}$ of saline; $6.8 \mathrm{~mm}$, i.m., i.p., or i.t.), NGF ( $1 \mu \mathrm{g}$ in $5 \mu \mathrm{l}$ of saline; $15 \mathrm{~mm}$, i.m.), GDNF ( $1 \mu \mathrm{g}$ in $5 \mu \mathrm{l}$ of saline; $8.4 \mathrm{~mm}$, i.m.), BDNF ( $1 \mu \mathrm{g}$ in $5 \mu \mathrm{l}$ of saline; $7.2 \mathrm{~mm}$, i.m.), control saline (i.m.), or control artificial CSF (i.t.) once every other day for the duration of 2 weeks (unless otherwise noted). Human recombinant NT-3, GDNF, BDNF, and mouse NGF $2.5 \mathrm{~S}$ were purchased from Chemicon International (Temecula, CA.) Trophic factors were dissolved in CSF ( $1 \mu \mathrm{g}$ in $5 \mu \mathrm{l}$ of CSF) for intrathecal injections and in saline ( $1 \mu \mathrm{g}$ in $5 \mu \mathrm{l}$ of saline) for intramuscular and intraperitoneal injections. Intrathecal injections were performed according to Hylden and Wilcox (1980) between the L6 and S1 vertebrae with a 27 gauge needle. Control animals received intramuscular injections of $5 \mu \mathrm{l}$ of saline or intrathecal injections of $5 \mu \mathrm{l}$ of CSF. All intramuscular injections were made into the right gastrocnemius muscle, and neurotrophins were delivered via a $10 \mu$ l Hamilton syringe (Hamilton, Reno, NV).

\section{Behavioral analyses}

Mechanical. Mice were tested for behavioral responses to mechanical stimuli using methods described previously (Christianson et al., 2003a). Mice were placed under bottomless plastic chambers $(3 \times 8 \times 12 \mathrm{~cm})$ on top of a wire mesh-top table and allowed to acclimate for $30 \mathrm{~min}$. Single calibrated von Frey monofilaments (Stoelting, Wood Dale, IL) were applied to the plantar surface of each foot. Responses to a single monofilament were recorded for each animal; the percentage response for each foot was obtained by determining the number of withdrawals in response to the different filaments (of five applications to each foot). Comparisons were made between test groups using one-way ANOVA and Fisher's protected least-significant differences (PLSD).

Thermal. Four weeks after acid injection, mice were tested for behavioral responses to thermal stimuli using methods described previously (Christianson et al., 2003a). Mice were placed under plastic bottomless chambers on a glass surface of a thermal analgesiometer (Department of
Anesthesiology, University of California, San Diego, La Jolla, CA) and allowed to acclimate for at least $30 \mathrm{~min}$ (Hargreaves et al., 1988; Dirig et al., 1997). An acute thermal stimulus from a high-intensity projector lamp bulb (CXL/CXR; 8V; 50W; Eiko, Tokaimura, Japan) was directed to the plantar surface of the hindpaw from beneath the glass surface. The stimulus lamp intensity was calibrated to 4.75 amperes before each testing session, and paw withdrawal latency was automatically measured to the nearest $0.01 \mathrm{sec}$. This stimulus intensity level produced a glassheating rate of $1.6^{\circ} \mathrm{C} / \mathrm{sec}$, which is within the range of selective C-fiber activation (Yeomans and Proudfit, 1996; Yeomans et al., 1996; Dirig et al., 1997). Tests were performed in triplicate, alternating between hindpaws to allow skin temperature to normalize before restimulation. The average latency was quantified for each animal and the average for each treatment group was compared using one-way ANOVA and Fisher's PLSD.

\section{Fos immunohistochemistry}

Wild-type and high-OE myo/NT-3 mice were injected with saline or acid on days 0 and 2 in the right gastrocnemius muscle. On days 5 or 16, all mice received a continuous, light palpation of the right hindpaw for 2 min (Honore et al., 2000). Two hours later, mice were deeply anesthetized with sodium pentobarbital $(65 \mathrm{mg} / \mathrm{kg}$, i.p.) and perfused intracardially with $0.1 \mathrm{~m}$ PBS, $\mathrm{pH} 7.4$, followed by $4 \%$ formaldehyde in $0.1 \mathrm{M}$ PBS, $\mathrm{pH}$ 7.4. The lumbar enlargement of the spinal cord was removed and nicked to identify right and left sides. The cord was postfixed for $2 \mathrm{hr}$ and cryoprotected overnight in 30\% sucrose. Transverse sections through the spinal cord were cut on a cryostat at $20 \mu \mathrm{m}$ and mounted on Superfrost microscope slides (Fisher Scientific, Chicago, IL). Immunocytochemistry was performed by blocking sections for $1 \mathrm{hr}$ in $1.5 \%$ normal goat serum, $0.5 \%$ porcine gelatin, and $0.2 \%$ Triton X-100 in Superblock buffer, pH 7.4 (Pierce, Rockford, IL). Sections were incubated overnight with a rabbit polyclonal Fos primary antibody (1:1000; Santa Cruz Biotechnology, Santa Cruz, CA) at $4^{\circ} \mathrm{C}$ under humidified conditions. The primary antibody was removed by two washes in PBS, and sections were then incubated with secondary antibody (donkey anti-rabbit CY3; 1:200 in $0.1 \mathrm{M}$ PBS; Jackson ImmunoResearch, West Grove, PA) for $1 \mathrm{hr}$ at $4^{\circ} \mathrm{C}$. Sections were rinsed and coverslipped before viewing. As a control, the primary antibody was omitted, resulting in no staining.

Six randomly selected slide sections $(\sim 80 \mu \mathrm{m}$ between sections) from each animal were coded to ensure the observer was blinded to the identity of the animal. The right and left sides of gray matter were photographed using a Nikon (Tokyo, Japan) E800 microscope attached to a Magnafire digital camera (Optronics, Goleta, CA). Using Adobe Photoshop (Adobe Systems, San Jose, CA), digital images were overlaid with a template to delineate spinal laminas, and immunopositive neurons in laminas I-II, III-IV, and V-VI were counted. Data from saline- or acid-injected wildtype or high-OE myo/NT-3 mice were compared using one-way ANOVA and Fisher's post hoc test for multiple comparisons.

\section{Results}

\section{Normal mechanical sensitivity of the paw is not altered by NT-3 overexpression}

The myogenin promoter was used to create transgenic mice that overexpress NT-3 in skeletal muscle and yielded different founder lines (Wright et al., 1997). Line \#5150 [highoverexpressing (OE)] maintains high levels of NT-3 overexpression in muscle postnatally, whereas line \#5160 carries the myo/ NT-3 transgene but expresses NT-3 in muscle near wild-type levels (low-OE) (Taylor et al., 2001).

To determine whether baseline differences existed among wild-type and myo/NT-3 mice in their response to mechanical stimuli, wild-type and transgenic littermates from high-OE myo/ $N T-3$ matings were examined for their responses to nine different von Frey monofilaments (Fig. 1). Because both transgenic lines had been bred onto a CF-1 background, responses of low-OE myo/NT-3 mice were compared with wild-type mice derived from high-OE myo/NT-3 litters. No differences were evident between high-OE myo/NT-3 $(n=7)$, low-OE myo/NT-3 $(n=7)$, 


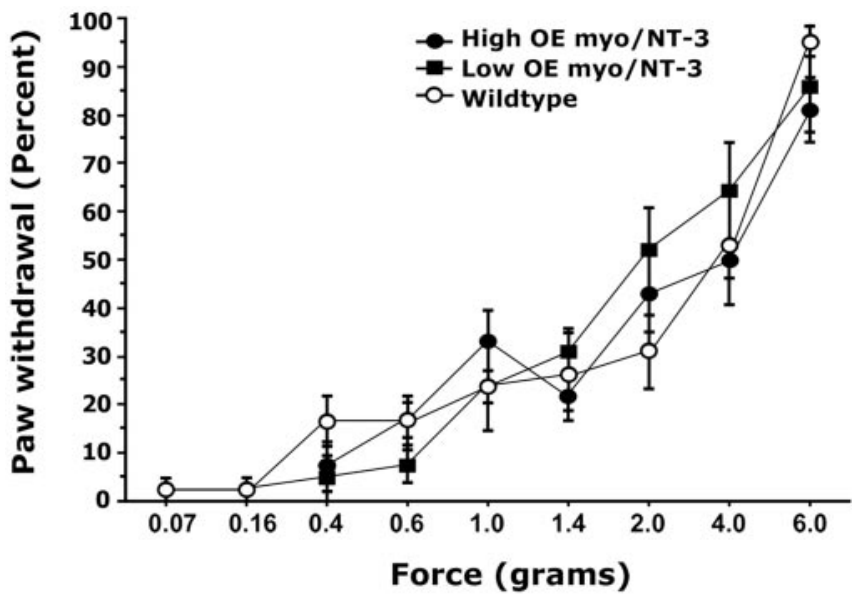

Figure 1. NT-3 overexpression does not alter baseline mechanical sensitivity of the hindpaw. Mechanical responsiveness of high-0E myo/NT-3, low-OE myo/NT-3, and wild-type mice was assessed using a series of nine different von Frey monofilaments. Data are plotted as percentage withdrawal responses from five applications to each foot (10 total for each monofilament). No significant differences were detected among the groups, revealing that the presence of the NT-3 transgene in low-0E myo/NT-3 mice or NT-3 overexpression in high-0E myo/ NT-3 mice do not alter mechanical sensitivity of the paw ( $p>0.05$; ANOVA). Error bars represent SEM.

and wild-type $(n=7)$ mice in their withdrawal responses to all von Frey monofilaments tested $(p>0.05)$. Similarly, no differences were detected among high-OE myo/NT-3, low-OE myo/ $N T$-3, or wild-type mice in their paw withdrawal thresholds to radiant heat applied to the hindpaw $(p>0.05)$ (Table 1$)$. These data demonstrate that NT-3 overexpression in skeletal muscle does not affect baseline responses to mechanical or thermal stimuli.

\section{Persistent acid-induced mechanical hyperalgesia fails to develop in high-OE myo/NT-3 mice}

To assess whether intramuscular acid injection results in hindlimb hyperalgesia similar to that produced in rats, and whether differences exist between wild-type and myo/NT-3 mice, animals received two injections of acidic saline, $2 \mathrm{~d}$ apart, in their right gastrocnemius muscle. Mice were then examined for their hindlimb withdrawal responses to three different von Frey monofilaments (Fig. 2). Within $1 \mathrm{~d}$ after the second acid injection, wildtype $(n=7)$ and low-OE myo/NT-3 $(n=7)$ mice developed a persistent hyperalgesia in response to mechanical stimuli. The mechanical hyperalgesia was clearly evident in the foot ipsilateral to the acid injection with all filaments tested (Fig. 2, left). Before the acid injection, wild-type mice withdrew at a rate of $48.6 \pm$ $10 \%$ in response to the 1.4 gm monofilament. However, $6 \mathrm{~d}$ after the second acid injection, the percentage response increased to $68.6 \pm 5.9 \%$. The contralateral foot also displayed significant hyperalgesia to each monofilament (Fig. 2, right). Similar to studies in rat, the increase in sensitivity to mechanical stimulus continued throughout the duration of the experiment (4 weeks), suggesting that it develops into a chronic hyperalgesia (Sluka et al., 2001). The responses of low-OE myo/NT-3 mice were identical to wild-type mice, consistent with the fact that they carry the myo/NT-3 transgene but do not overexpress NT-3 in skeletal muscle (Fig. 2).

In comparison, high-OE myo/NT-3 $(n=7)$ mice developed a transient hyperalgesia after the second acid injection that was evident in both paws (Fig. 2). However, withdrawal responses returned to preinjection levels within 2-3 d after the second in-
Table 1. Thermal latencies to radiant heat

\begin{tabular}{lll}
\hline & Ipsilateral paw & Contralateral paw \\
\hline $\begin{array}{ll}\text { NT-3 overexpression } \\
\text { Wild type }\end{array}$ & \\
Low-OE myo/NT-3 & $6.07 \pm 0.58$ & $7.39 \pm 0.71$ \\
High-0E myo/NT-3 & $7.35 \pm 0.73$ & $9.93 \pm 1.1$ \\
Exogenous NT-3 treatment & $7.12 \pm 0.70$ & $8.75 \pm 1.3$ \\
WT-no acid, untreated & & \\
WT-saline treated (i.m.) & $4.43 \pm 0.40$ & $4.51 \pm 0.25$ \\
WT-NT-3 treated (i.m.) & $5.01 \pm 0.32$ & $5.11 \pm 0.41$ \\
Routes of administration & $4.45 \pm 0.29$ & $5.22 \pm 0.57$ \\
WT-NT-3 treated (i.m.) & & \\
WT-NT-3 treated (i.p.) & $3.96 \pm 0.76$ & $3.41 \pm 0.58$ \\
WT-NT-3 treated (i.t.) & $3.40 \pm 0.63$ & $4.01+0.46$ \\
\hline
\end{tabular}

Four weeks after acid injection, thermal responses were recorded from each paw as withdrawal latencies from a radiant heat source (seconds). In regard to NT-3 overexpression, wild-type (WT) mice were derived from litters within the myo/NT-3 colony. For exogenous treatments, wild-type CF-1 mice were purchased from Charles River. Comparisons were made between test groups using one-way ANOVA and Fisher's PLSD. No differences in thermal thresholds were observed among transgenic or NT-3-treated mice or by using different routes of NT-3 administration $(p>0.05)$. Data are plotted as means \pm SEM

jection in both paws. The reversal of the hyperalgesia in high-OE myo/NT-3 mice was evident in both ipsilateral and contralateral feet for each monofilament (Fig. 2, left and right), suggesting that overexpression of NT-3 in muscle can reverse mechanisms that lead to chronic, acid-induced mechanical hyperalgesia.

The initial report describing the effects of acid injection demonstrated that thermal responses were not altered after intramuscular acid injection (Sluka et al., 2001). Here, hindlimb withdrawal thresholds were also tested in wild-type, low-OE, and high-OE myo/NT-3 mice 4 weeks after acid injection. No significant differences in thermal withdrawal thresholds were detected among wild-type, low-OE, and high-OE myo/NT-3 mice in either the ipsilateral or contralateral paw $(p>0.05)$ (Table 1).

\section{Exogenous NT-3 treatment also attenuates mechanical hyperalgesia}

To assess whether the reduction in mechanical hyperalgesia resulted from developmental changes in the peripheral nervous system in high-OE myo/NT-3 mice or rather the presence of intramuscular NT-3, wild-type CF-1 mice were treated intramuscularly with two different doses of exogenous NT-3. Mice were injected with NT-3 into the same gastrocnemius every other day starting with the first acid injection and were compared with mice that received vehicle only (Fig. 3). Both acid-injected and acidinjected, saline-treated mice developed persistent mechanical hyperalgesia after the second acid injection.

Mice that received exogenous NT-3 ( $1 \mu$ g every other day; $n=$ 7) developed a transient mechanical hyperalgesia after the second acid injection. However, percentage withdrawal responses began to return to baseline levels within 3-4 d after the acid injections (Fig. 3). It interesting to note that reduction of the mechanical hyperalgesia took longer in NT-3-treated mice compared with high-OE myo/NT-3 mice. The mechanical withdrawal responses of NT-3-treated mice reached baseline levels by 1 week after the second acid injection, and the protective effect of NT-3 was evident in both paws for each monofilament (Fig. 3). A 10-fold lower dose of NT-3 ( $0.1 \mu \mathrm{g}$ every other day; $n=6)$ failed to reverse the mechanical hyperalgesia (data not shown). These results suggest that exogenous NT-3 delivered intramuscularly reproduces the protection observed in high-OE myo/NT-3 mice.

The capacity of NT-3 to reverse the mechanical hyperalgesia evoked by acid injection was compared with the related family members NGF and BDNF and to the TGF $\beta$ family member 
GDNF. A separate group of acid-injected CF-1 mice was injected intramuscularly with exogenous NGF $(n=7)$ (Fig. 3$)$. In contrast to NT-3, NGF had no effect on the development or maintenance of the mechanical hyperalgesia after acid injection (Fig. 3). Finally, additional groups of acidinjected mice were injected intramuscularly with exogenous BDNF or GDNF ( $n=$ 6 each) (Fig. 4). Similar to NGF, neither BDNF nor GDNF had any significant effect on the acid-induced hyperalgesia (Fig. 4). These doses of NGF and GDNF have been shown to alter cutaneous innervation and/or behavioral responses to cutaneous stimuli (Christianson and Wright, 2003; Christianson et al., 2003a,b), suggesting that the absence of effects by NGF and GDNF was not attributable to inadequate dosing.

Responses to noxious thermal stimuli were also tested in mice receiving NT-3. No significant differences in thermal thresholds were detected among acidinjected, acid-injected plus vehicletreated, and acid-injected plus NT-3treated mice in either the ipsilateral or contralateral paw $(p>0.05)$ (Table 1$)$. The lack of change of thermal thresholds in acid-injected and acid-injected plus vehicle mice is consistent with reports that acid injection does not alter thermal sensitivities (Sluka et al., 2001).

To rule out whether exogenous NT-3, by itself, altered baseline mechanical sensitivity of the paw, non-acid-injected mice were treated intramuscularly with exogenous NT-3. Non-acid-injected CF-1 mice were treated intramuscularly with exogenous NT-3 ( $1 \mu \mathrm{g} / \mathrm{d}$ every other day) for $8 \mathrm{~d}$. Treatment with NT-3 had no significant effect on withdrawal responses to a $1.4 \mathrm{gm}$ von Frey monofilament (day 1, $34.7 \pm 2.9 \%$; day $6,36.7 \pm 4.2 \% ; p>$ $0.05)$, demonstrating that intramuscular NT-3 treatment itself had no effect on mechanical sensitivity of the hindpaw.

\section{Intramuscular NT-3 treatment}

To address whether the actions of NT-3 were contingent on target-derived access, intramuscular NT-3 administration was compared with intrathecal and intraperitoneal NT-3 administration. Acid-injected mice were treated with NT-3 intraperitoneally, intrathecally, or intramuscularly, and the mechanical sensitivity of the hindpaw to a 1.4 gm monofilament was compared with acidinjected mice treated intramuscularly with vehicle (Fig. 5). As expected, mice treated with NT-3 intramuscularly developed a transient hyperalgesia that returned to baseline levels within $4 \mathrm{~d}$ after the second acid injection. In contrast, mice treated with NT-3 intraperitoneally or intrathecally responded to mechanical stimuli similar to acid-injected control mice ( $p>0.05$ ) (Fig. 5), demonstrating that the effects of NT-3 were only potent when introduced via the target (i.e., muscle).

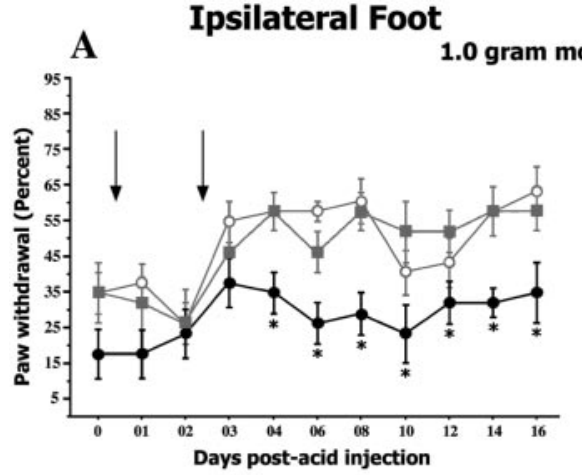

Contralateral Foot

B

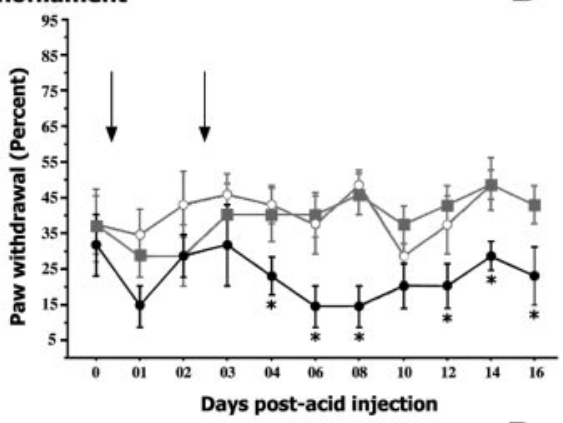

D
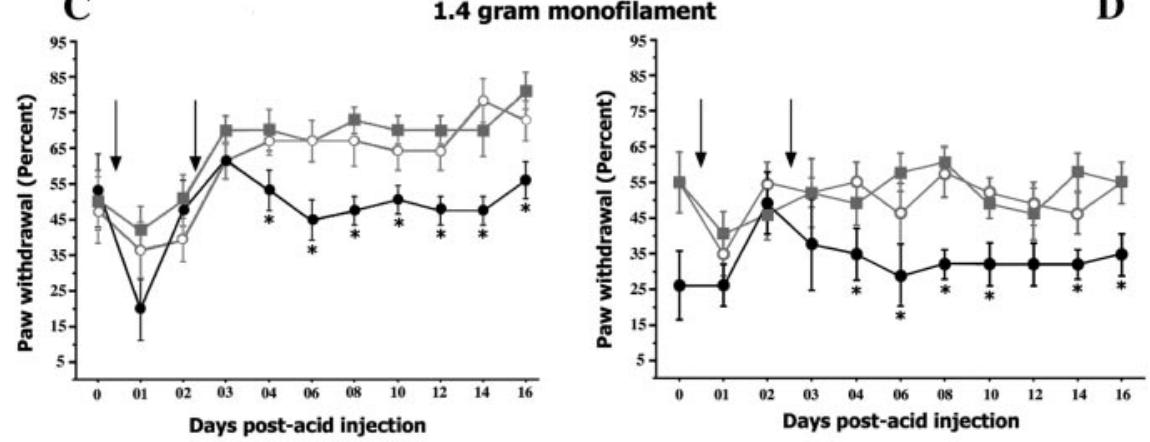

F
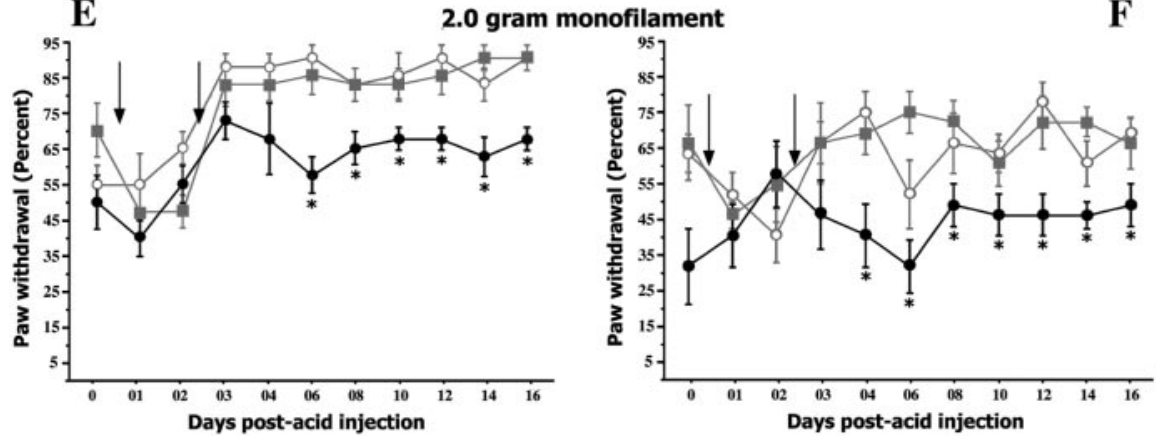

Figure 2. NT-3 overexpression prevents the development of acid-induced chronic mechanical hyperalgesia. Mechanical responsiveness of high-0E myo/NT-3, low-OE myo/NT-3, and wild-type mice in response to acid injection into the gastrocnemius (gray-filled squares) mice. Three different monofilaments $[1.0 \mathrm{gm}(A, B), 1.4 \mathrm{gm}(C, D)$, and $2.0 \mathrm{gm}(E, F)]$ were applied to the (left) and contralateral (right) hindpaws. Wild-type and low-OE myo/NT-3 mice develop long-lasting increased sensi列 significantly different. ${ }^{*}$ denotes significant difference between high-0E myo/NT-3 and wild-type mice ( $p<0.05$; ANOVA; Fisher's PLSD). Error bars represent SEM.

\section{Neurotrophic protection only occurs during the initiation of hyperalgesia}

In all experiments thus far, NT-3 treatment began concurrently with the first acid injection and continued until the termination of the experiment. To assess whether NT-3 treatment could reverse the acid-injected hyperalgesia at subsequent stages or after the emergence of the hyperalgesia, acid-injected mice received NT-3 treatment (i.m.) $2 \mathrm{~d}(n=6)$ or $4 \mathrm{~d}(n=6)$ after the first acid injection (Fig. 6). Mice receiving NT-3 treatment on day 2 after first acid injection displayed progressively reduced paw withdrawal responses to a 1.4 gm monofilament (day 1, $36.1 \pm 5.1 \%$; day $6,53.0 \pm 6.1 \%$ ) (Fig. 6). However, mice receiving NT-3 treatment on day 4 after the first acid injection continued to display mechanical hyperalgesia (day $1,33.3 \pm 8.6 \%$; day 6 , $63.1 \pm 5.6 \%$ ) (Fig. 6). 


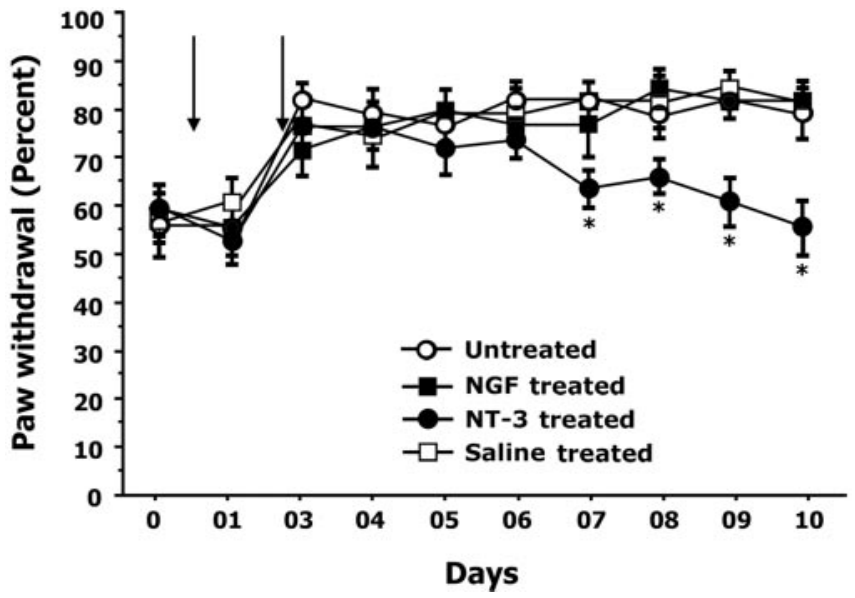

Figure 3. Exogenous NT-3 treatment attenuates acid-induced mechanical hyperalgesia. Graph illustrates percentage withdrawal responses to a $1.4 \mathrm{gm}$ monofilament after acid injection in untreated wild-type mice (open circles) compared with mice treated intramuscularly with exogenous NT-3 (black-filled circles), NGF (black-filled squares), or vehicle (saline; open squares). Arrows indicate days on which mice received acid injections into the gastrocnemius. Mice were treated with $1 \mu \mathrm{g}$ of NT-3 or NGF in $5 \mu$ l of saline every other day beginning on the day of the first acid injection. Compared with NGF-treated mice, saline-treated mice, and untreated wild-type mice, NT-3-treated mice show reduced mechanical hyperalgesia $4 \mathrm{~d}$ after the second acid injection. NGF had no effect on acid-induced mechanical hyperalgesia. * denotes significant difference between NT-3-treated and nontreated wild-type mice ( $p<0.05$; ANOVA; Fisher's PLSD). Error bars represent SEM.

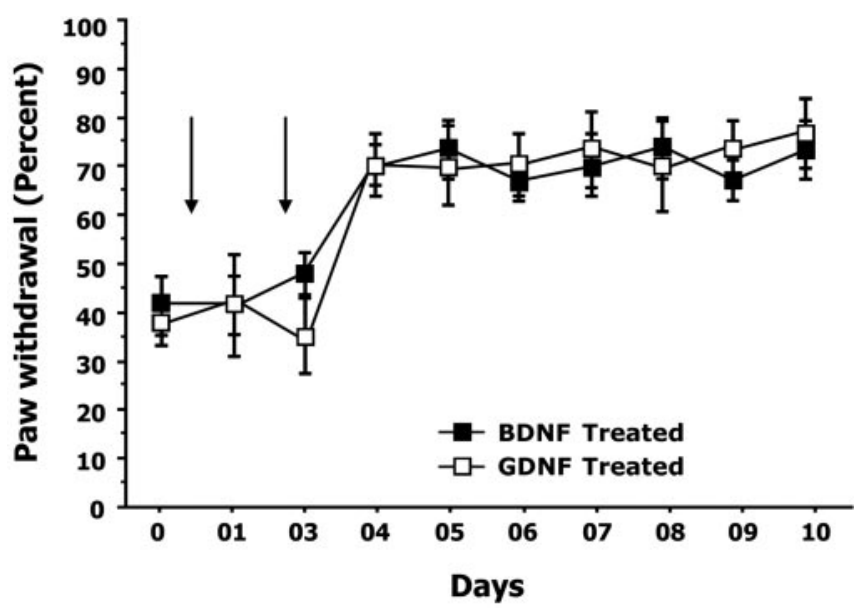

Figure 4. Exogenous GDNF and BDNF treatment do not attenuate acid-induced mechanical hyperalgesia. Graph illustrates percentage withdrawal responses to a $1.4 \mathrm{gm}$ monofilament after acid injection in wild-type mice treated intramuscularly with exogenous BDNF (black-filled squares) or GDNF (open squares). Arrows indicate days on which mice received acid injections into the gastrocnemius. Mice were treated with $1 \mu \mathrm{g}$ of GDNF or BDNF in $5 \mu$ l of saline every other day beginning on the day of the first acid injection. GDNF-treated and BDNF-treated mice failed to show any reduction in mechanical hyperalgesia after acid injection. Error bars represent SEM.

\section{The actions of NT-3 appear permanent}

Acid-injected mice treated with intramuscular NT-3 (Fig. 5) were used to determine whether the hyperalgesia would reappear after the termination of NT-3 treatment. After the cessation of NT-3 treatment on day 10 , mechanical sensitivity of the acid-injected wild-type mice was followed for an additional $7 \mathrm{~d}$. As shown in Figure 7 , the percentage of withdrawal responses to a $1.4 \mathrm{gm}$ monofilament displayed by these mice was similar to levels observed before acid injection, suggesting that NT-3 treatment permanently reversed the effects of acid injection on mechanical sensitivity.

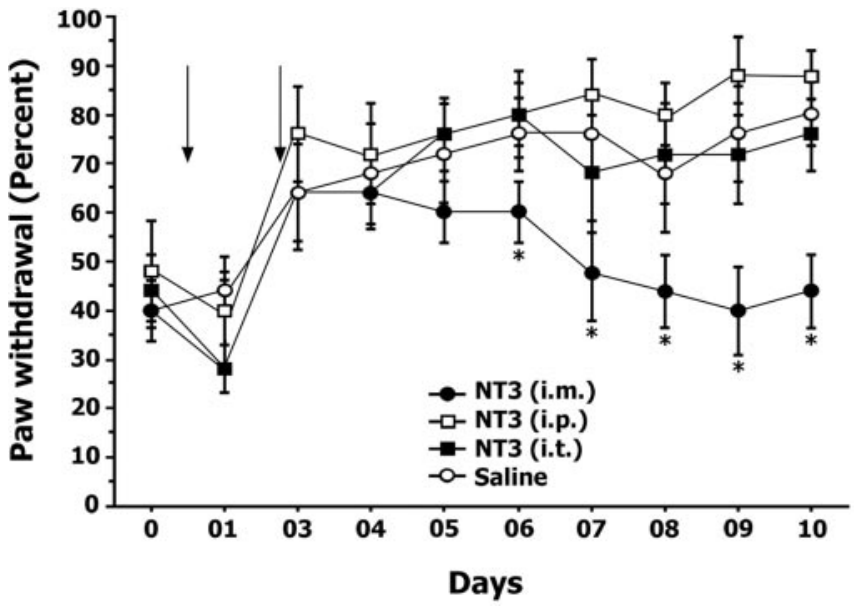

Figure 5. Intramuscular NT-3 treatment is the most effective route of administration. Exogenous NT-3 was administered via different routes: intramuscular, intraperitoneal, or intrathecal. Graph illustrates percentage withdrawal responses to a $1.4 \mathrm{gm}$ monofilament after acid injection. Arrows indicate days on which intramuscular acid injections were administered. Only mice treated with exogenous NT-3 via intramuscular route showed a reduction in mechanical hyperalgesia when compared with mice treated with NT-3 via intraperitoneal and intrathecal routes and mice treated with saline intramuscularly. ${ }^{*}$ denotes significant difference between intramuscularly NT-3-treated and saline-treated wild-type mice ( $p<0.05$; ANOVA; Fisher's PLSD). Error bars represent SEM.

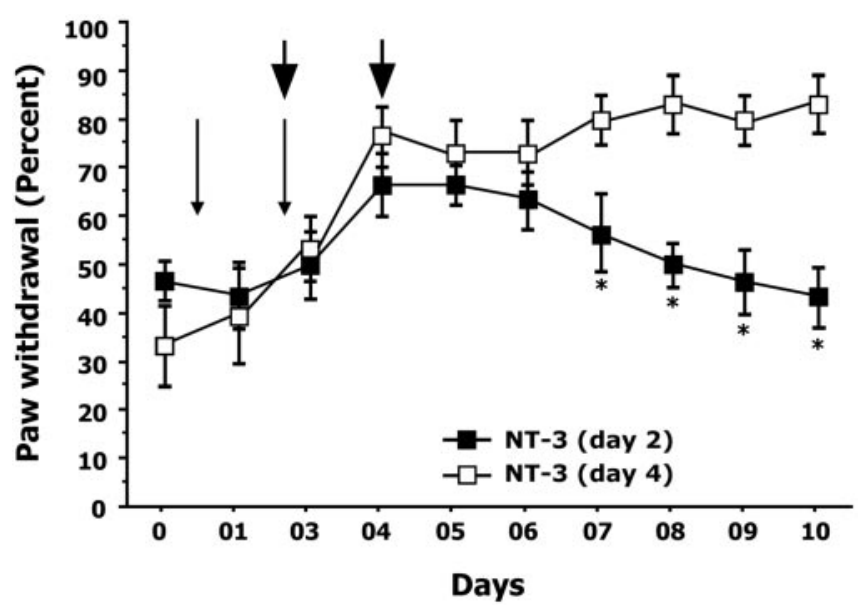

Figure 6. NT-3 treatment is required during the initial stages of the development of hyperalgesia. To determine whether a critical period exists for the neuroprotective actions of NT-3, intramuscular treatments (denoted by arrowheads) were initiated on the day of the second acid injection (day 2) or $2 \mathrm{~d}$ after the second acid injection (day 4) and continued every other day. Graph illustrates percentage withdrawal responses to a $1.4 \mathrm{gm}$ monofilament after acid injection. Thin arrows indicate days on which intramuscular acid injections were administered. Mice treated with NT-3 on day 2 displayed a significant reduction in mechanical hyperalgesia from day 7 onwards. In contrast, mice treated with NT-3 beginning on day 4 failed to show a reduction in hyperalgesia, suggesting that NT-3 administration is required during the initial stages (day 2) of the development of hyperalgesia. * denotes significant difference between NT-3treated (day 2) and NT-3-treated (day 4) mice ( $p<0.05$; ANOVA; Fisher's PLSD). Error bars represent SEM.

\section{Acid-induced increase in spinal cord Fos expression is} blocked by NT-3

Experiments were performed to determine whether Fos expression in the spinal cord mirrored the acid-induced hindpaw hyperalgesia and whether NT-3 reduced Fos expression. Immunocytochemistry for Fos expression was performed on L4/L5 spinal segments in wild-type or high-OE myo/NT-3 littermates 5 or $16 \mathrm{~d}$ after injection of saline or acid ( $n=3$ for each group) in the 


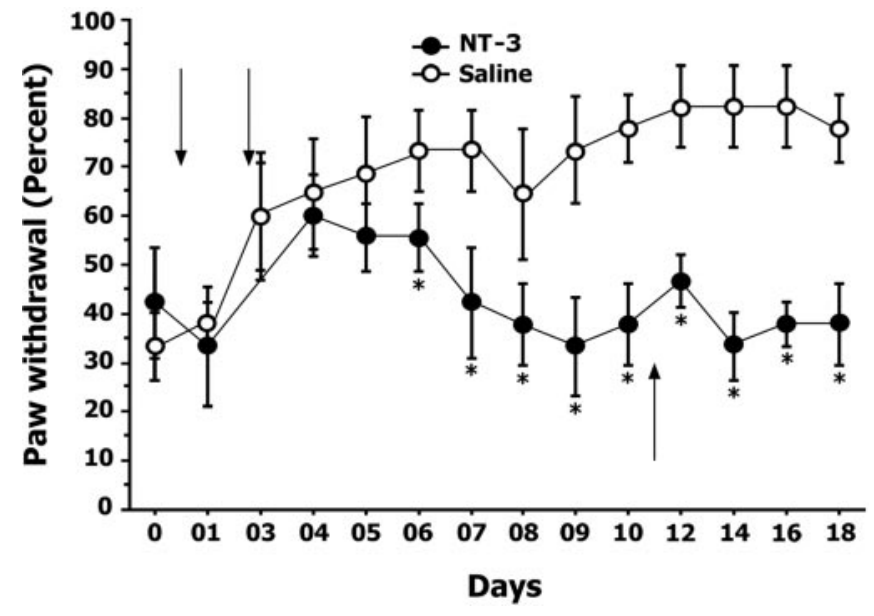

Figure 7. The neuroprotective actions of NT-3 appear permanent. To determine whether cessation of NT-3 treatment would result in a return to increased paw sensitivity, NT-3 treatment was stopped from day 11 onwards (indicated by upward arrow) in mice that were treated with NT-3 (i.m.) beginning on the first day of acid injection. Graph illustrates percentage withdrawal responses to a $1.4 \mathrm{gm}$ monofilament after acid injection. Downward arrows indicate days on which intramuscular acid injections were administered. After cessation of NT-3 treatment, mice continued to exhibit reduced mechanical hyperalgesia after treatment of NT-3 compared with saline-treated mice. * denotes significant differences between NT-3-treated and saline-treated mice ( $p<0.05$; ANOVA; Fisher's PLSD). Error bars represent SEM.

gastrocnemius. In all cases, Fos expression was quantified in both contralateral and ipsilateral sides of the spinal cord in relation to the palpated foot. Initial experiments revealed that acid injection in either wild-type or high-OE myo/NT-3 mice had no effect on Fos expression in the spinal cord (data not shown). Therefore, Fos expression was induced by light palpation of the foot on the injected side before perfusion (Honore et al., 2000). Using this approach, acid injection in wild-type mice resulted in a significant increase in the number of Fos-positive neurons on the ipsilateral side of the cord at both time points in all laminas examined (Tables 2, 3; Fig. 8). The largest increase was observed in lamina I-II, where 10-15 strongly positive cells were visible in the superficial aspect of the medial dorsal horn, a region that receives input from distal axons that innervate the foot. No differences were observed on the contralateral side (Tables 2, 3).

Analysis of high-OE myo/NT-3 mice revealed a minor increase in the number of Fos-positive neurons in the spinal cord compared with wild-type littermates, particularly in the deeper laminas. This increase may be related to the large number of NT-3-responsive Ia spindle afferent fibers in these mice, leading to higher baseline levels of Fos expression (Wright et al., 1997). However, acid injection of high-OE myo/NT-3 mice did not stimulate Fos expression in any laminas after foot palpation (Table 2, Fig. 8).

To test whether increased Fos expression would reappear after the cessation of NT-3 treatment, groups of mice were acid injected and NT-3 treated for $10 \mathrm{~d}$. Control mice only received acid injections and no NT-3 treatment. Six days after the termination of NT-3 treatment, Fos expression was assessed $2 \mathrm{hr}$ after right hindpaw palpation. As shown in Table 3, heightened Fos expression did not return after the termination of NT-3 treatment. These results are consistent with the absence of heightened mechanical sensitivity to von Frey monofilament application after the cessation of NT-3 treatment.

\section{Discussion}

Acid injection into the gastrocnemius produces a chronic mechanical hyperalgesia of the hindpaw. The increased sensitivity is interpreted as a secondary hyperalgesia, because it extends beyond the injected area and includes both limbs (Sluka et al., 2001). Here, we demonstrate that NT-3 can prevent chronic secondary hyperalgesia induced by acid. These neuroprotective actions appear unique to NT-3, because other neurotrophic factors failed to elicit similar effects. The potency of NT-3 is highest when accessed via neurons innervating the muscle, and the reversing actions appear permanent. These data suggest that NT-3responsive muscle afferents play a critical role in the initiation of acid-induced mechanical hyperalgesia, and NT-3 may be effective in modulating neuropathic pain originating from muscle.

\section{Muscle-derived mechanical secondary hyperalgesia}

Pain arising from musculoskeletal origin is associated with mechanical hyperalgesia, and the intramuscular acid model used in this study is proposed to mimic aspects of musculoskeletal pain associated with secondary hyperalgesia (Sluka et al., 2001). Low $\mathrm{pH}$ can elicit pain, and decreases in tissue $\mathrm{pH}$ occur in a number of biological settings (Hood et al., 1988; Pan et al., 1988; Issberner et al., 1996; Reeh and Steen, 1996). In humans, intramuscular infusion of acidic buffer causes painful sensations and increased sensitivity to mechanical stimulation (Steen and Reeh, 1993; Issberner et al., 1996). Secondary hyperalgesia can also be elicited by intramuscular injections of inflammatory agents such as carrageenan (Radhakrishnan et al., 2003). It will be important to determine whether NT-3 has neuroprotective actions on other forms of muscle-induced pain.

Convergent input in the spinal cord from muscle-paw fibers and receptive field plasticity likely underlie the acid-induced secondary hyperalgesia (Treede et al., 1992). The hindpaw and the gastrocnemius are innervated by different sensory fibers but are located in the same dermatome and myotome, respectively (Greene, 1963). Although muscle afferents are clearly involved in the initiation of intramuscular acid-induced blockade of muscle, afferent activity after acid injection suggests that continued muscle afferent input is not required (Sluka et al., 2001). The acid does not cause muscle damage, and the requirement of two acid injections suggests that these sequences alter central processing (Sluka et al., 2001). These spinal alterations involve NMDA and non-NMDA receptors and are sensitive to opioid analgesics (Skyba et al., 2002; Sluka et al., 2002).

It appears that ASIC3 channels are critical for the initiation of acid-induced hyperalgesia (Sluka et al., 2003). ASIC3 is a protongated sodium channel expressed by DRG neurons (Waldmann et al., 1997; Garciá-Añoveros et al., 2001; Voilley et al., 2001; Sluka et al., 2003). After acid injection, wide dynamic range neurons in the dorsal horn increase their responsiveness to mechanical stimuli and expand their receptive fields to include the contralateral paw. ASIC3 null mice do not develop acid-induced hyperalgesia and do not display changes in wide dynamic range activity (Sluka et al., 2003). Thus, it is proposed that acid-induced secondary hyperalgesia is mediated by alterations in wide dynamic range neurons triggered by activation of ASIC3-expressing muscle afferents. Our results now focus this view to NT-3-responsive muscle afferents.

\section{Neurotrophins in chronic pain}

There is strong evidence that neurotrophins play an important role in the pathophysiology of neuropathic pain and can slow or 
Table 2. Fos expression in the spinal cord $5 \mathrm{~d}$ after acid injection

\begin{tabular}{|c|c|c|c|c|c|c|}
\hline & \multicolumn{2}{|l|}{ Wild type } & \multicolumn{2}{|c|}{ High-0E myo/NT-3 } & \multicolumn{2}{|c|}{ Wild type NT-3 treated } \\
\hline & Contralateral & Ipsilateral & Contralateral & Ipsilateral & Contralateral & Ipsilateral \\
\hline \multicolumn{7}{|c|}{ Lamina I-II } \\
\hline Acid & $1.73 \pm 0.64$ & $14.87 \pm 1.05^{*}$ & $3.07 \pm 1.88$ & $3.07 \pm 1.20^{* *}$ & $1.0 \pm 0.32$ & $1.2 \pm 0.49$ \\
\hline \multicolumn{7}{|c|}{ Lamina III-IV } \\
\hline Saline & $3.07 \pm 0.84$ & $3.60 \pm 0.53$ & $9.40 \pm 5.20$ & $9.73 \pm 5.25$ & & \\
\hline Acid & $5.27 \pm 0.74$ & $12.93 \pm 1.73^{*}$ & $7.87 \pm 3.99$ & $8.27 \pm 2.97$ & $1.2 \pm 0.37$ & $1.4 \pm 0.51$ \\
\hline Acid & $12.53 \pm 1.27$ & $23.00 \pm 1.21^{*}$ & $15.93 \pm 7.53$ & $17.87 \pm 5.97$ & $1.0 \pm 0.55$ & $0.6 \pm 0.24$ \\
\hline
\end{tabular}

Table 3. Fos expression in spinal cord $16 \mathrm{~d}$ after acid injection

\begin{tabular}{lllll}
\hline & Wild type & & \multicolumn{2}{l}{ Wild type NT-3 treated } \\
\cline { 2 - 3 } \cline { 5 - 5 } & Contralateral & Ipsilateral & Contralateral & Ipsilateral \\
\hline Lamina I-II & $3.8 \pm 0.86$ & $10.6 \pm 0.93^{*}$ & $1.25 \pm 0.35$ & $3.45 \pm 0.42$ \\
Lamina III-IV & $4.6 \pm 0.68$ & $11.8 \pm 0.58^{*}$ & $1.80 \pm 0.49$ & $3.55 \pm 0.73$ \\
Lamina V-VI & $5.0 \pm 1.05$ & $7.8 \pm 1.07^{*}$ & $2.60 \pm 0.62$ & $3.65 \pm 0.39$ \\
\hline
\end{tabular}

The number of Fos-positive cells was counted from different spinal laminas from both ipsilateral and contralateral sides of the spinal cord $16 \mathrm{~d}$ after acid injection. Comparisons were made between groups using one-way ANOVA and Fisher's PLSD. In wild-type mice, acid injection caused a significant increase in the number of Fos-positive neurons compared with wild-type mice receiving NT-3 treatment for $10 \mathrm{~d}$ in each lamina ( ${ }^{*} p<0.05$ ). Fos expression in wild-type NT-3-treated mice confirmed that cessation of NT-3 treatment $10 \mathrm{~d}$ after the initial acid injection correlates with a lack of Fos activity 1 week later. Data are plotted as means \pm SEM; $n=3$ animals per group.
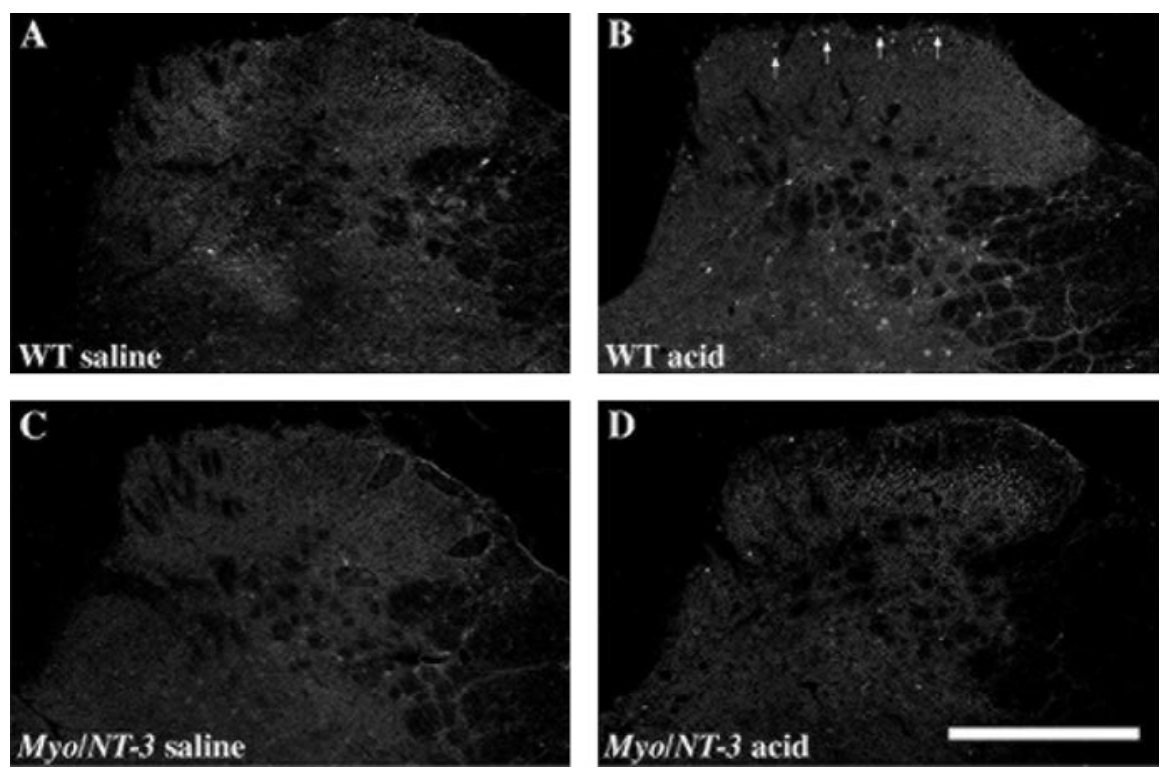

Figure 8. Fos expression in the lumbar (L5) dorsal horn $5 \mathrm{~d}$ after initial acid injection. Photomicrographs of Fos expression in representative sections from the ipsilateral mouse spinal cord $5 \mathrm{~d}$ after acid injection. $A$, Section from a wild-type (WT) mouse injected with saline illustrating few Fos-positive cells. B, Five days after acid injection, wild-type mice displayed significantly more Fos-positive cells in each lamina. This increase in acid-injected mice is consistent with their increase in mechanical sensitivity to von Frey monofilaments. Arrows denote cells within laminas I-II that are strongly positive for Fos. C, Saline injection in high- $0 \mathrm{E}$ myo/NT-3 mice resulted in no significant differences in Fos expression. D, Acid injection in high-0E myo/NT-3 mice failed to increase Fos expression, similar to wild-type mice. Scale bar, $200 \mu \mathrm{m}$.

stop disease progression (Sah et al., 2003). NGF plays a role in peripheral inflammation and has been tested in clinical trial to alleviate pain with moderate success (Apfel et al., 1998; McArthur et al., 2000). BDNF is released by nociceptive afferents in the dorsal horn and is a modulator of central sensitization (Thompson et al., 1999; Pezet et al., 2002). GDNF can prevent the development of pain and phenotypic changes in neurons after nerve injury (Hao et al., 1998;
Boucher et al., 2000). Recent studies have begun to report beneficial actions of NT-3 related to neuropathic pain. A single systemic dose of NT-3 in rats induced mechanical hypoalgesia $1 \mathrm{~d}$ after NT-3 injection. In the same study, NT-3 administered systemically for 2 weeks significantly reduced electrically evoked release of substance $P$ from the spinal cord, suggesting possible anti-nociceptive actions of NT-3 involving substance P (Malcangio et al., 1997). In addition, intrathecal administration of NT-3 to rats receiving a chronic constriction injury (CCI) to the sciatic nerve blocks the development of thermal but not mechanical hyperalgesia. NT-3 also reduced the injury-induced upregulation of the vanilloid receptor VR-1 in the DRG and spinal cord that normally occurs after CCI (Wilson-Gerwing and Verge, 2003). Together with our findings, these studies have begun to identify circumstances in which NT-3 plays a role in modulating nociceptive signaling.

\section{Neuroprotective actions of NT-3}

Neurotrophins are well known for their developmental actions mediated via retrograde transport to the soma. The inability of high-OE myo/NT-3 mice to develop mechanical hyperalgesia, coupled with the fact that only intramuscular administration of NT-3 could block the hyperalgesia, suggests that target-derived NT-3 may provide a critical signal to muscle afferent neurons. Alternatively, intramuscular administration may have provided the highest concentration of NT-3 to axons. Systemic NT-3 injection has a very short half-life (Poduslo and Curran, 1996), and it is plausible that 
NT-3 delivered intramuscularly might be protected within the muscle compared with other routes. This is important in light of the use of neurotrophins in clinical trials. These results suggest that the actions of NT-3 occur locally out at the peripheral terminal or require retrograde transport from the periphery. If the actions of NT-3 are attributable to penetration and half-life, a long lasting activator of TrkC should have similar effects when administered either intramuscularly or intrathecally. Alternatively, if NT-3 actions are related to retrograde transport, anchoring NT-3 to a substrate that activates the receptor but prevents internalization and retrograde transport should shed light on these mechanisms.

The intramuscular acid paradigm requires two injections within $2-5 \mathrm{~d}$ to elicit the mechanical hypersensitivity, and the second injection plays a critical role in triggering the development of the hyperalgesia. Here, NT-3 treatment was only effective if provided at the time of the second injection, suggesting that the actions of NT-3 are critical during initial stages of hyperalgesia. It is important to note that exogenous NT-3 treatment was successful in preventing the hyperalgesia, suggesting that the effects were not attributable to the altered proprioceptive system in myo/ NT-3 mice (Wright et al., 1997). The delay in the return of mechanical thresholds in NT-3-treated mice may be caused by the difference in exposure to NT-3 levels. High-OE myo/NT-3 transgenic mice likely provide a constant source of NT-3 to muscle afferents, whereas levels from exogenous NT-3 administration vary as a result of its rapid degradation. Finally, experiments that continued to follow acid-injected mice after the cessation of NT-3 treatment demonstrated no return of paw hypersensitivity, supporting the view that actions of NT-3 were attributable to mechanisms that prevented the onset of chronic hypersensitivity.

\section{Selectivity of neurotrophin-3}

Several neurotrophins were tested to determine whether the neuroprotective actions were unique to NT-3; injections of NGF, BDNF, or GDNF failed to suppress the acid-induced paw hypersensitivity. These results support the view that the actions of NT-3 were selective and not caused by general trophic effects mediated through other Trks or p75. It is known that NT-3 can mediate certain effects by activating the NGF receptor TrkA (Belliveau et al., 1997; Gratto and Verge, 2003). Also, these results suggest that the fiber type(s) responsible for triggering the chronic mechanical hyperalgesia are NT-3-responsive muscle afferents. Moreover, the doses of neurotrophins administered are effective in models of diabetic neuropathy, suggesting that the absence of effects by NGF, GDNF, and BDNF were not to attributable to inadequate dosing (Christianson and Wright, 2003; Christianson et al., 2003a,b).

\section{Suppression of acid-induced Fos expression by NT-3}

Fos is a biomarker of neuronal activation in response to peripheral stimuli, and spinal Fos expression strongly correlates with pain intensity (for review, see Harris, 1998; Bon et al., 2002). Demonstration that acid injection increases Fos expression after paw palpation is consistent with the role of Fos in pain signaling and corroborates our behavioral data. It is important to note that acid injection itself did not induce Fos expression but required mechanical stimulation of the paw. The finding that Fos expression was not increased in acid-injected myo/NT-3 mice and wildtype mice treated with NT-3 parallels the ability of NT-3 to reverse the mechanical hyperalgesia using behavioral assessments. It is interesting that both control and acid-injected myo/NT-3 mice had increased Fos expression in deeper laminas. It is plau- sible to suggest that this increase is related to the large number of proprioceptive afferents that survive in response to NT-3 overexpression, resulting in increased baseline spinal activity (Wright et al., 1997). Our results with Fos expression suggest that NT-3 blocks or reverses acid-induced changes in muscle afferents that prevent alterations in Fos activity. Moreover, heightened Fos expression did not reappear after the cessation of NT-3 treatment in wild-type mice.

\section{References}

Apfel SC, Kessler JA, Adornato BT, Litchy WJ, Sanders C, Rask CA (1998) Recombinant human nerve growth factor in the treatment of diabetic polyneuropathy. NGF Study Group. Neurology 51:695-702.

Belliveau DJ, Krivko I, Kohn J, Lachance C, Pozniak C, Rusakov D, Kaplan D, Miller FD (1997) NGF and neurotrophin-3 both activate TrkA on sympathetic neurons but differentially regulate survival and neuritogenesis. J Cell Biol 136:375-388.

Bon K, Wilson SG, Mogil JS, Roberts WJ (2002) Genetic evidence for the correlation of deep dorsal horn Fos protein immunoreactivity with tonic formalin pain behavior. J Pain 3:181-189.

Boucher TJ, Okuse K, Bennett DL, Munson JB, Wood JN, McMahon SB (2000) Potent analgesic effects of GDNF in neuropathic pain states. Science 290:124-127.

Christianson JA, Wright DE (2003) NGF and NT-3 treatments improve select aspects of large-fiber diabetic neuropathy in mice. Soc Neurosci Abstr 29:478.9.

Christianson JA, Riekhof JT, Wright DE (2003a) Restorative effects of neurotrophin treatment on diabetes-induced cutaneous axon loss in mice. Exp Neurol 179:188-199.

Christianson JA, Ryals JM, McCarson KE, Wright DE (2003b) Beneficial actions of neurotrophin treatment on diabetes-induced hypoalgesia in mice. J Pain 4:493-504..

Dirig DM, Salami A, Rathbun ML, Ozaki GT, Yaksh TL (1997) Characterization of variables defining hind paw withdrawal latency evoked by radiant thermal stimuli. J Neurosci Methods 76:183-191.

Fyffe REW (1992) Laminar organization of primary afferent terminations in the mammalian spinal cord. In: Sensory neurons: diversity, development and plasticity (Lawson SN, ed), pp 131-142. New York: Oxford UP.

Garciá-Añoveros J, Samad TA, Zuvela-Jelaska L, Woolf CJ, Corey DP (2001) Transport and localization of the DEG/ENaC ion channel $\mathrm{BNaC} 1 \alpha$ to peripheral mechanosensory terminals of dorsal root ganglia neurons. J Neurosci 21:2678-2686.

Gratto KA, Verge VM (2003) Neurotrophin-3 down-regulates trkA mRNA, NGF high-affinity binding sites, and associated phenotype in adult DRG neurons. Eur J Neurosci 18:1535-1548.

Graven-Nielsen T, Mense S (2001) The peripheral apparatus of muscle pain: evidence from animal and human studies. Clin J Pain 17:2-10.

Greene EC (1963) Nervous system. In: Anatomy of the rat, Vol XXVII, Chap 6, pp 115-175. New York: Hafner.

Hao S, Mata M, Wolfe D, Huang S, Glorioso JC, Fink DJ (1998) HSVmediated gene transfer of the glial cell-derived neurotrophic factor provides an antiallodynic effect on neuropathic pain. Mol Ther 8:367-375.

Hargreaves K, Dubner R, Brown F, Flores C, Joris J (1988) A new and sensitive method for measuring thermal nociception in cutaneous hyperalgesia. Pain 32:77-88.

Harris J (1998) Using c-fos as a neural marker of pain. Brain Res Bull 45:1-8.

Hoeger-Bement MK, Sluka KA (2003) Phosphorylation of CREB and mechanical hyperalgesia is reversed by blockade of the cAMP pathway in a time-dependent manner after repeated intramuscular acid injections. J Neurosci 23:5437-5445.

Honore P, Rogers SD, Schwei MJ, Salak-Johnson JL, Luger NM, Sabino MC, Clohisy DR, Mantyh PW (2000) Murine models of inflammatory, neuropathic and cancer pain each generate a unique set of neurochemical changes in the spinal cord and sensory neurons. Neuroscience 98:585598.

Hood VL, Schubert C, Keller U, Muller S (1988) Effect of systemic pH on $\mathrm{pHi}$ and lactic acid generation in exhaustive forearm exercise. Am J Physiol 255:F479-F485.

Hylden JL, Wilcox GL (1980) Intrathecal morphine in mice: a new technique. Eur J Pharmacol 67:313-316. 
Issberner U, Reeh PW, Steen KH (1996) Pain due to tissue acidosis: a mechanism for inflammatory and ischemic myalgia? Neurosci Lett 208:191-194.

Kehl LJ, Trempe TM, Hargreaves KM (2000) A new animal model for assessing mechanisms and management of muscle hyperalgesia. Pain $85: 333-343$

Lawson SN (1992) Morphological and biochemical cell types of sensory neurons. In: Sensory neurons: diversity, development and plasticity (Lawson SN, ed), pp 27-59. New York: Oxford UP.

Malcangio M, Garrett NE, Cruwys S, Tomlinson DR (1997) Nerve growth factor and neurotrophin-3-induced changes in nociceptive threshold and the release of substance $\mathrm{P}$ from the rat isolated spinal cord. J Neurosci 17:8459-8467.

McArthur JC, Yiannoutsos C, Simpson DM, Adornato BT, Singer EJ, Hollander H, Marra C, Rubin M, Cohen BA, Tucker T, Navia BA, Schifitto G, Katzenstein D, Rask C, Zaborski L, Smith ME, Shriver S, Millar L, Clifford DB, Karalnik IJ (2000) A phase II trial of nerve growth factor for sensory neuropathy associated with HIV infection. AIDS Clinical Trials Group Team 291. Neurology 54:1080-1088.

McMahon SB, Armanini MP, Ling LH, Phillips HS (1994) Expression and coexpression of Trk receptors in subpopulations of adult primary sensory neurons projecting to identified peripheral targets. Neuron 12:1161-1171.

Molliver DC, Wright DE, Leitner ML, Parsadanian AS, Doster K, Wen D, Yan Q, Snider WD (1997) IB4-binding DRG neurons switch from NGF to GDNF dependence in early postnatal life. Neuron 19:849-861.

Nagano M, Suzuki H (2003) Quantitative analyses of expression of GDNF and neurotrophins during postnatal development in rat skeletal muscle. Neurosci Res 45:391-399.

Pan JW, Hamm JR, Rothman DL, Shulman RG (1988) Intracellular pH in human skeletal muscle by 1H NMR. Proc Natl Acad Sci USA 85:7836-7839.

Pezet S, Malcangio M, McMahon SB (2002) BDNF: a neuromodulator in nociceptive pathways? Brain Res Brain Res Rev 40:240-249.

Poduslo JF, Curran GL (1996) Permeability at the blood-brain and bloodnerve barriers of the neurotrophic factors: NGF, CNTF, NT-3, BDNF. Brain Res Mol Brain Res 36:280-286.

Price MP, McIlwrath SL, Xie J, Cheng C, Qiao J, Tarr DE, Sluka KA, Brennan TJ, Lewin GR, Welsh MJ (2001) The DRASIC cation channel contributes to the detection of cutaneous touch and acid stimuli in mice. Neuron 32:1071-1083.

Radhakrishnan R, Moore SA, Sluka KA (2003) Unilateral carrageenan injection into muscle or joint induces chronic bilateral hyperalgesia in rats. Pain 104:567-577.

Reeh PW, Steen KH (1996) Tissue acidosis in nociception and pain. Prog Brain Res 113:143-151.

Sah DW, Ossipov MH, Porreca F (2003) Neurotrophic factors as novel therapeutics for neuropathic pain. Nat Rev Drug Discov 2:460-472.

Schafers M, Sorkin LS, Sommer C (2003) Intramuscular injection of tumor necrosis factor-alpha induces muscle hyperalgesia in rats. Pain 104:579-588.
Skyba DA, King EW, Sluka KA (2002) Effects of NMDA and non-NMDA ionotropic glutamate receptor antagonists on the development and maintenance of hyperalgesia induced by repeated intramuscular injection of acidic saline. Pain 98:69-78

Sluka KA, Kalra A, Moore SA (2001) Unilateral intramuscular injections of acidic saline produce a bilateral, long-lasting hyperalgesia. Muscle Nerve 24:37-46.

Sluka KA, Rohlwing JJ, Bussey RA, Eikenberry SA, Wilken JM (2002) Chronic muscle pain induced by repeated acid injection is reversed by spinally administered mu- and delta-, but not kappa-, opioid receptor agonists. J Pharmacol Exp Ther 302:1146-1150.

Sluka KA, Price MP, Breese NM, Stucky CL, Wemmie JA, Welsh MJ (2003) Chronic hyperalgesia induced by repeated acid injections in muscle is abolished by the loss of ASIC3, but not ASIC1. Pain 106:229-239.

Snider WD (1994) Functions of the neurotrophins during nervous system development: what the knockouts are teaching us. Cell 77:627-638.

Steen KH, Reeh PW (1993) Sustained graded pain and hyperalgesia from harmless experimental tissue acidosis in human skin. Neurosci Lett 154:113-116.

Taylor MD, Vancura R, Patterson CL, Williams JM, Riekhof JT, Wright DE (2001) Postnatal regulation of limb proprioception by muscle-derived neurotrophin-3. J Comp Neurol 432:244-258.

Thompson SW, Bennett DL, Kerr BJ, Bradbury EJ, McMahon SB (1999) Brain-derived neurotrophic factor is an endogenous modulator of nociceptive responses in the spinal cord. Proc Natl Acad Sci USA 96:7714-7718

Treede RD, Meyer RA, Raja SN, Campbell JN (1992) Peripheral and central mechanisms of cutaneous hyperalgesia. Prog Neurobiol 38:397-421.

Voilley N, de Weille J, Mamet J, Lazdunski M (2001) Nonsteroid antiinflammatory drugs inhibit both the activity and the inflammationinduced expression of acid-sensing ion channels in nociceptors. J Neurosci 21:8026-8033.

Waldmann R, Champigny G, Bassilana F, Heurteaux C, Lazdunski M (1997) A proton-gated cation channel involved in acid-sensing. Nature 386:173-177.

Wilson-Gerwing TD, Verge VMK (2003) NT-3 attenuation of thermal hyperalgesia is associated with decreased VR-1 expression. Soc Neurosci Abstr 29:381.15.

Wright DE, Zhou L, Kucera J, Snider WD (1997) Introduction of a neurotrophin-3 transgene into muscle selectively rescues proprioceptive neurons in mice lacking endogenous neurotrophin-3. Neuron 19:503-517.

Yeomans DC, Proudfit HK (1996) Nociceptive responses to high and low rates of noxious cutaneous heating are mediated by different nociceptors in the rat: electrophysiological evidence. Pain 68:141-150.

Yeomans DC, Pirec V, Proudfit HK (1996) Nociceptive responses to high and low rates of noxious cutaneous heating are mediated by different nociceptors in the rat: behavioral evidence. Pain 68:133-140. 